\title{
Neonatal Antidepressant Exposure has Lasting Effects on Behavior and Serotonin Circuitry
}

\author{
Dorota Maciag ${ }^{1,4}$, Kimberly L Simpson ${ }^{2,4}$, David Coppinger ${ }^{3}$, Yuefeng Lu $^{2}$, Yue Wang ${ }^{2}$, Rick CS Lin ${ }^{2}$ \\ and Ian A Paul*,1,3 \\ 'Department of Psychiatry \& Human Behavior, University of Mississippi Medical Center, Jackson, MS, USA; ${ }^{2}$ Department of Anatomy, University \\ of Mississippi Medical Center, Jackson, MS, USA; ${ }^{3}$ Department of Pharmacology \& Toxicology, University of Mississippi Medical Center, Jackson, \\ MS, USA
}

\begin{abstract}
A significant fraction of infants born to mothers taking selective serotonin reuptake inhibitors (SSRIs) during late pregnancy display clear signs of antidepressant withdrawal indicating that these drugs can penetrate fetal brain in utero at biologically significant levels. Previous studies in rodents have demonstrated that early exposure to some antidepressants can result in persistent abnormalities in adult behavior and indices of monoaminergic activity. Here, we show that chronic neonatal (postnatal days 8-2I) exposure to citalopram (5 mg/kg, twice daily, s.c.), a potent and highly selective SSRI, results in profound reductions in both the rate-limiting serotonin synthetic enzyme (tryptophan hydroxylase) in dorsal raphe and in serotonin transporter expression in cortex that persist into adulthood. Furthermore, neonatal exposure to citalopram produces selective changes in behavior in adult rats including increased locomotor activity and decreased sexual behavior similar to that previously reported for antidepressants that are nonselective monoamine transport inhibitors. These data indicate that the previously reported neurobehavioral effects of antidepressants are a consequence of their effects on the serotonin transporter. Moreover, these data argue that exposure to SSRIs at an early age can disrupt the normal maturation of the serotonin system and alter serotonin-dependent neuronal processes. It is not known whether this effect of SSRIs is paralleled in humans; however, these data suggest that in utero, exposure to SSRIs may have unforeseen long-term neurobehavioral consequences. Neuropsychopharmacology (2006) 3 I, 47-57. doi: I 0. I038/sj.npp. I 300823; published online I 3 July 2005
\end{abstract}

Keywords: neonatal exposure; citalopram; clomipramine; tryptophan hydroxylase; serotonin transporter; behavior

\section{INTRODUCTION}

Selective serotonin reuptake inhibitors (SSRIs) are the pharmacological treatment of choice for depression, anxiety, and obsessive-compulsive disorder. Moreover, these drugs are specifically recommended for treatment of these disorders during pregnancy and lactation (Wisner et al, 2000; Cohen et al, 2004). This is largely due to their low perceived toxicity to both mother and fetus or infant. As a consequence, there is a significant and increasing likelihood that children will be exposed to SSRIs either in utero or via breast milk. The long-term consequences of this early exposure to SSRIs are largely unknown.

A large fraction of children exposed in utero to SSRIs have been reported to display signs of antidepressant

\footnotetext{
*Correspondence: Dr IA Paul, Department of Psychiatry and Human Behavior, University of Mississippi Medical Center, 2500 North State St, Jackson, MS 392I6-4505, USA, Tel: 60I 984 5883, Fax: 60I 984 5884, E-mail: ipaul@psychiatry.umsmed.edu

${ }^{4}$ These authors contributed equally to this work.

Received 10 February 2005; revised 9 May 2005; accepted 3 June 2005 Online publication: 9 June 2005 at http://www.acnp.org/citations/ Npp060905050093/default.pdf
}

withdrawal in the first week or two of life (Nordeng et al, 2001; Laine et al, 2003; Zeskind and Stephens, 2004; Sanz et al, 2005), indicating that the fetus can be exposed to neurobiologically relevant doses of these drugs. In fact, significant SSRI and metabolite concentrations have been detected in both umbilical cord blood and amniotic fluid in women taking these medications during pregnancy. The mean ratios of umbilical cord to maternal serum concentrations ranged from 0.29 to 0.89 (Hostetter et al, 2000; Hendrick et al, 2003).

Children exposed to SSRIs in utero have been followed for up to 72 months postnatally and have not been reported to display increased behavioral abnormalities compared to unexposed children although they have been reported to display subtle changes in motor development and in motor movement control (Costei et al, 2002; Nulman et al, 2002; Casper et al, 2003). However, no study has followed such children beyond early childhood. Thus, it remains possible that such exposure may result in later neurobehavioral abnormalities.

In neonatal rodents, chronic administration of serotonin reuptake inhibitors (clomipramine, fluoxetine, zimeldine, LU-10-134C) as well as some other tricyclic antidepressants 
but not the atypical antidepressants iprindole or nomifensine during the early life period from postnatal day 8 (PN8) to PN21 results in a pattern of maladaptive behaviors that are evident long after drug discontinuation and persist into adulthood (Mirmiran et al, 1981; Hilakivi et al, 1984; Hilakivi and Hilakivi, 1987; Hansen et al, 1997; Ansorge et al, 2004). These behavioral changes, described here as the 'neonatal antidepressant exposure syndrome (NADES)', in rats include alterations in locomotor activity, reduced male sexual activity and competence, increased ethanol consumption, dysregulation of the hypothalamic-pituitaryadrenal axis, increased rapid eye movement (REM) sleep time and reduced latency to enter the REM sleep phase, and increased immobility in the forced swim test (Mirmiran et al, 1981; Hilakivi et al, 1984; Hilakivi and Hilakivi, 1987; Hartley et al, 1990; Hansen et al, 1997). In contrast, adults exposed to similar doses and durations of antidepressants exhibit no persistent behavioral effects after drug discontinuation, indicating that the neurobiological response to long-term antidepressant administration differs markedly between early life and adulthood.

Similarly, serotonin transporter (SERT) knockout mice have been reported to display increased anxiety- and depression-like behaviors, reduced aggressive behavior, and exaggerated reponse to environmental stress (Holmes et al, 2003; Lira et al, 2003; Ansorge et al, 2004). Moreover, there is evidence from these studies that the absence of SERT expression is accompanied by reductions in the density of $5 \mathrm{HT}_{1 \mathrm{~A}}$ receptors in dorsal raphe (DR) as well as increases in the density of $5 \mathrm{HT}_{2 \mathrm{~A}}$ and $5 \mathrm{HT}_{2 \mathrm{C}}$ receptors in amygdala and choroid plexus (Li et al, 2000, 2003). However, it is not known whether these effects are the result of adult adaptation to the absence of SERT or are a function of alterations in extracellular serotonin levels during critical periods of development. Nonetheless, it might be predicted from these studies that disruption of SERT function by SSRIs would be accompanied by alterations in aggressive and conflict-related behaviors.

Brain growth (mass) in rodents begins antenatally, peaks at 5-10 days postnatally (PN5-PN10) and continues to PN21-PN28 days with adult mass attained after that time. By comparison, human brain growth begins antenatally, peaks at birth and continues beyond 30 months postnatally (Dobbing, 1974). In addition to simple increases in brain mass, synaptogenesis and glial proliferation begin during late antenatal periods and continue through postnatal day (PN)40 in the rat (4-6 years old in humans). Likewise, neurotransmitter receptor development continues well into adolescence in both rats and humans (PN50 + in rats, 1217 years old in humans). For both rat and human, the competence of the blood-brain barrier is not mature until well after birth. Thus, the developing CNS during the prenatal and early postnatal periods is uniquely vulnerable to neurobiological teratogenicity due to exogenous drug exposure (Lauder, 1990; Whitaker-Azmitia, 1991).

In the rat, monoaminergic neurons appear relatively early in prenatal development (10-14 days of gestation) (Lauder and Bloom, 1974). Synaptogenesis, while beginning in late prenatal development, undergoes its most extensive development between postnatal days 5-20 (Lauder and Bloom, 1975). This is accompanied by increases in synthetic enzyme (tyrosine and tryptophan hydroxylase) activity up to PN30-PN40 (Johnston and Coyle, 1980). Likewise, monoaminergic transporter proteins are poorly expressed prenatally, but undergo a massive proliferation and subsequent 'pruning' in the 5-6 weeks after birth (Hansson et al, 1998; Zhou et al, 2000). Thus in the rat, extensive development of neurotransmitter systems affected by antidepressant treatments occurs up to 5-6 weeks postnatally. It therefore appears that the NADES paradigm in rats closely parallels the exposure of children to antidepressants during the late stages of in utero development and the first 3 years of postnatal life.

The neurobiological events that produce NADES are unknown. The paradigm was introduced by Mirmiran et al (1981) using clomipramine as a pharmacological means of suppressing active sleep during development and this group first reported that neonatal clomipramine exposure resulted in reductions in cortical and medullary weight, total protein, and total DNA (Mirmiran et al, 1983). Since these initial studies, a number of investigators have reported that neonatal clomipramine exposure results in reductions in basal monoamine concentration and turnover in subcortical regions (striatum, hypothalamus, limbic structures) (Hilakivi et al, 1987a; Feenstra et al, 1996; Vijayakumar and Meti, 1999; Yannielli et al, 1999). Similarly, animals exposed to clomipramine neonatally display reduced firing of neurons in the DR nuclei (Yavari et al, 1993; Kinney et al, 1997). Finally, two groups have reported that neonatal clomipramine impairs hypothalamic-pituitary axis responsiveness as marked by increased circulating levels of corticosterone and reduced suppression of corticosterone secretion in response to dexamethasone treatment (Ogawa et al, 1994; Prathiba et al, 1998). However, the mechanism(s) by which neonatal but not adult antidepressant exposure produce these effects is/are unknown.

Serotonergic cell bodies in the raphe nuclei project throughout the CNS. Since the neonatal treatment period associated with NADES coincides with the period of rapid development of monoaminergic systems, it is reasonable to suspect that alterations in normal synaptic concentrations of serotonin during critical periods of neuronal interaction would have far-reaching consequences throughout the CNS. By changing the performance of cognitive, sensory, and motor circuits, such alterations would be expected to distort the way information is received, interpreted, and acted upon. Xu et al (2004) have provided an anatomical basis for this assertion by showing that neonatal administration of the SSRI, paroxetine, disrupts the organization of barrel field cortex via interference with the refinement of thalamocortical afferents.

In fact, the early genesis of the central monoaminergic neurons in mammals has repeatedly led to the postulation of a trophic role of monoamines on brain morphogenesis. Serotonin is one of the first neurotransmitters to appear in the CNS and has been proposed to act as a developmental signal in cell proliferation, differentiation, and apoptosis (Lauder, 1990; Azmitia, 2001; Verney et al, 2002). Tryptophan hydroxylase (TPH) is the rate-limiting enzyme in the production of serotonin, and during development, brain serotonin levels and TPH mRNA increases 35 -fold in DR between embryonic day 18 and PN22 in rat (Rind et al, 2000). Likewise, the level of SERT mRNA, the primary means of removing serotonin from the synapse and 
terminating serotonin signaling, has been found to be related to synaptogenesis (Ivgy-May et al, 1994; Hansson et al, 1998). In human brain, serotonergic neurons have been detected by 5 weeks of gestation and synaptic levels of serotonin increase during the first 2 years after birth and then decrease to adult levels after the age of 5 years (Sodhi and Sanders-Bush, 2004).

Given that the development of maladaptive behaviors after early life exposure to antidepressants is seen in animals and humans alike, we hypothesized that transient SSRI treatment alters the long-term chemical profile of the raphe cortical projection system. In order to reveal cellular substrates of serotonergic dysfunction, which may correlate with stereotyped behavioral repertoires of NADES rats, we examined TPH and SERT immunoreactivity in male rats at PN22 and PN130 after they had received neonatal antidepressant treatment (PN8-PN21). For these studies, we employed the highly selective SSRI, citalopram, and clomipramine, a tricyclic compound that preferentially inhibits serotonin reuptake and is the antidepressant first reported to induce NADES.

\section{MATERIALS AND METHODS}

\section{Design}

The male offspring of timed-pregnant Long Evans rats were used in these experiments. All procedures were approved by the UMMC Animal Care and Use Committee and complied with AAALAC and NIH standards. Shortly after delivery (PN1-3), the pups were sexed and the males selected and crossfostered as necessary to produce litters of 4-5 pups. Female pups were euthanized. On PN6-7, pups were tattooed for identification. Beginning at PN8, rat pups were injected subcutaneously with citalopram (Tocris, Ellisville, $\mathrm{MO}$ ) at a dose $5 \mathrm{mg} / \mathrm{kg}$, clomipramine (Sigma-Aldrich, St Louis, MO) at a dose $15 \mathrm{mg} / \mathrm{kg}$, or saline in a volume of $0.1 \mathrm{ml}$ twice daily (0600 and 1200 hours) from PN8-PN21. Each litter included at least one pup in each treatment group. At PN21, $3 \mathrm{~h}$ after the last drug injection, five rats from the citalopram and clomipramine treatment groups were randomly chosen and killed in order to determine the serum and brain drug concentrations achieved in our exposure paradigm. At PN22, 16-24h after the last drug injection, four animals from each group were randomly chosen for perfusion and immunostaining. The others were weaned at PN28 and housed in groups of 2-3/cage under standard laboratory conditions with ad lib access to food and water. Except for weekly weighing, rats were left undisturbed until PN60.

\section{Behavioral Testing}

Behavioral testing was conducted on adult rats $(\geqslant \mathrm{PN} 60)$ during the dark phase of the light: dark cycle. Rats were brought to a sound-attenuated testing room to acclimate for $1 \mathrm{~h}$ before each test.

Locomotor activity. Rats were placed individually into locomotor activity-monitoring units (transparent Plexiglas, $43 \mathrm{~cm}^{2}$ floor, $20 \mathrm{~cm}$ walls-Opto-Varimex, Columbus Instruments) under moderate light conditions (300lux) for $30 \mathrm{~min}$. Four monitoring units were arranged in parallel so that at least one rat from each exposure group was recorded in each observation period. A computer acquisition system recorded horizontal and vertical activity in 5 min epochs. Data were analyzed for time locomoting, zone of activity, distance traveled, stereotypies, and rearing.

Sexual behavior. At PN90, each male rat was tested for sexual behavior. Males were placed in a clear Plexiglas observation chamber $(45 \times 25 \times 20 \mathrm{~cm})$ for a 10-min adaptation period. The test was initiated by placing a female into the arena with the male. A group of ovariectomized females (stimulus females) were brought into estrous with estradiol benzoate $(5 \mu \mathrm{g}$ s.c., 48 and $24 \mathrm{~h}$ prior to testing) and progesterone $(500 \mu \mathrm{g}$ s.c. $4-6 \mathrm{~h}$ prior to testing). Each test lasted $60 \mathrm{~min}$ and was conducted under dim red light. Each encounter was videotaped and analyzed for number of mounts, intromissions, ejaculations, latency to first mount, and latency to first intromission.

\section{Drug Concentration}

Rats ( $n=5$ /exposure group) were killed by decapitation $3 \mathrm{~h}$ after the last of 14 days drug exposure and samples of trunk blood and forebrain were collected. The brain samples were rapidly frozen on solid $\mathrm{CO}_{2}$. The blood samples were coagulated and centrifuged $(10000 \mathrm{~g}, 5 \mathrm{~min})$ and the serum aspirated and frozen on solid $\mathrm{CO}_{2}$. Coded samples were shipped to National Medical Services (Willow Park, PA) for gas chromatographic analysis of citalopram, clomipramine, and its metabolite desclomipramine. Analytic methods were adjusted for rat serum and brain matrices (Pel-Freez, Rogers, AR).

\section{TPH, SERT, and Neuron-Specific Nuclear Protein (NeuN) Immunocytochemistry}

At PN22 or PN130, rats were deeply anesthetized with sodium pentobarbital $(60-80 \mathrm{mg} / \mathrm{kg}$, i.p.) and perfused through the heart with saline followed by $3.5 \%$ paraformaldehyde in $0.1 \mathrm{M}$ phosphate-buffered saline at $\mathrm{pH}$ 7.4. Brains were removed immediately and sectioned the next day on a freezing microtome to a thickness of $40-80 \mu \mathrm{m}$.

Three sections with one cortical column from each side of the hemispheres from each case were examined. Changes in the chemical content of the dorsal raphe or cortical projection system were examined by fluorescence immunohistochemistry. More specifically, the expression of TPH was evaluated in DR cell bodies, and the expression of SERT was evaluated in axon terminals of the medial prefrontal and primary somatosensory cortices. Tissue was incubated overnight at $4{ }^{\circ} \mathrm{C}$ in TPH affinity-purified antiserum raised in sheep $(1: 1000-3000$; Chemicon International, Temecula, CA) or in polyclonal SERT antisera (1:1000-2000, a gift from Dr FC Zhou, Indiana University, Indianapolis, IN or Chemicon). To ensure that observed changes (ie decreases in number and intensity of immunoreactive cells) were attributable strictly to downregulation of chemical expression and not to cell death, the NeuN (neuron specific nuclear) staining procedure was performed using a rabbit polyclonal antiserum (dilution 1:200-500; Chemicon). NeuN labels neuronal cell bodies and permits neuronal 
losses to be assessed. After exposing to biotinylated antisheep or rabbit IgG (1:100; Vector, Burlingame, CA; ABC kit), samples were rinsed and treated with streptavidinconjugated Cy2 (green - NeuN) or Cy3 (red-TPH, SERT), (1:200-300; Jackson ImmunoResearch Laboratories, West Grove, PA).

Data analysis. Tissue from six subgroups of rats was immunohistochemically processed for TPH and SERT; four subgroups contained tissue from subjects 22 days of age, and two subgroups contained samples from adults. Each subgroup contained three to five brainstem or cortical sections from one saline-, one citalopram-, and one clomipramine-treated animal. Sections were marked for identification and processed simultaneously in the same reaction chamber. Staining patterns from each treatment were initially assessed on a subgroup by subgroup basis; differences were then averaged across subgroups with similarly aged and treated subjects. In particular, a Nikon E800 epifluorescent microscope was used to survey: (1) the number of cells in DR that express TPH, (2) the staining intensity of individual TPH-positive cells in DR, and (3) the density of cortical SERT-immunoreactive fiber networks. An imaging system equipped with a Photometrics Coolsnap ES CCD camera (Roper Scientific) and Metamorph imaging software (Universal Imaging Systems) was used to quantify data. More specifically, fiber density was calculated for each PN22 subgroup by acquiring images from three sections per case. Exposure times were kept constant for each series of tissue. Data were collected from two cortical columns at intervals that spanned layers I, II/III, and V/VI. The area of tissue occupied by immunoreactive fibers was measured by thresholding within a range of gray values that corresponded to labeled profiles. The percentage thresholded area was determined for each $\sim 100 \times 100 \mu \mathrm{m}^{2}$ microscopic field and averaged across individual lamina as well as cumulatively across superficial and deep layers. Final values were established by representing immunoreactive fiber density as a function of percentage thresholded area in saline-treated subjects.

\section{Statistical Analysis}

Data analyses were performed using SPSS software (Version 11.5, Chicago, IL). Statistical significance of the results was determined with single factor analysis of variance (ANOVA), followed by LSD test (locomotor activity and sexual behavior); Kaplan-Meier analysis using Log-Rank statistic (sexual behavior); single factor (drug exposure) repeated measures (days) ANOVA (body weight) and MannWhitney $U$-test (immunoreactivity).

\section{RESULTS}

\section{Locomotor Activity}

Locomotor activity was robustly increased in citalopramand clomipramine-treated rats when compared to control (saline). An increase in distance traveled $\left(\mathrm{F}_{2,14}=9.27\right.$, $P=0.003)$ and ambulatory time $\left(\mathrm{F}_{2,14}=6.79, P=0.009\right)$ was observed in both groups (Figure la and c). In the case of citalopram, this effect was most pronounced in the initial
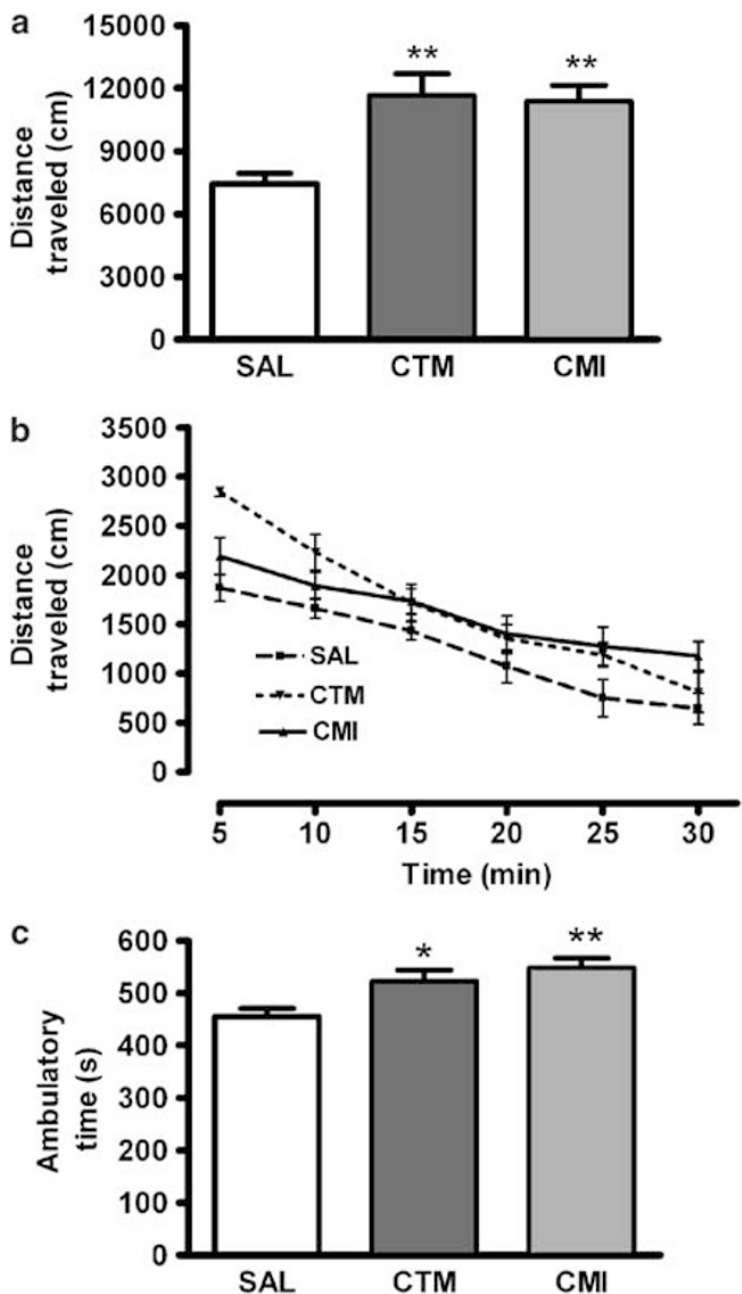

Figure I Effect of neonatal exposure to saline (SAL; $n=6$ ), citalopram $(\mathrm{CTM} ; n=5)$, and clomipramine (CMl; $n=6)$ on locomotor activity: $(a, b)$ distance traveled; (c) ambulatory time. Data represent the mean \pm SEM Single factor analysis of variance (ANOVA) followed by LSD test (** $P<0.0$ I; * $P<0.05$ vs $\mathrm{SAL}$ ).

period suggesting an exaggerated response to a novel environment, but was evident throughout the 30-min recording period (Figure $1 b$ ).

\section{Sexual Behavior}

Compared to saline-treated rats, rats neonatally exposed to citalopram and clomipramine exhibited lower sexual activity. Both citalopram (Log-Rank $=10.26, \mathrm{df}=1, P=0.0014)$ and clomipramine (Log-Rank $=4.37, \mathrm{df}=1, P=0.0365$ ) significantly impaired mounting behavior (Figure 2a). Similarly, these animals tended to display fewer numbers of intromissions $\left(\mathrm{F}_{2,14}=2.68, P=0.103\right)$ and ejaculations $\left(\mathrm{F}_{2,14}=3.17, P=0.073\right)$. However, a significant effect was observed only in CTM-exposed rats (Figure 2b). CTM- and CMI-treated rats also had longer intromission latency than SAL rats, but the differences were not significant (data not shown). 

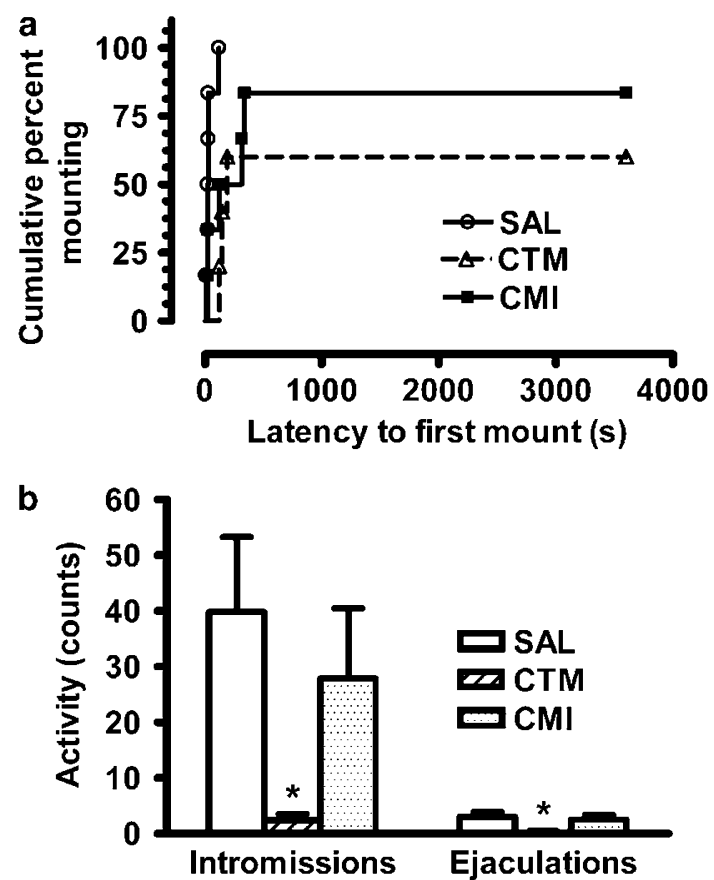

Figure 2 Effect of neonatal exposure to saline (SAL; $n=6)$, citalopram (CTM; $n=5)$, and clomipramine (CMl; $n=6$ ) on male sexual behavior: (a) Kaplan-Meier survival analysis of mounting behavior using Log-Rank statistic, $\mathrm{SAL}$ vs $\mathrm{CTM}=10.26, \quad P=0.0014 ; \quad \mathrm{SAL}$ vs $\mathrm{CMI}=4.37$, $P=0.0365$. (b) Number of intromissions and ejaculations; ANOVA followed by LSD test (*P $<0.05$ vs SAL).

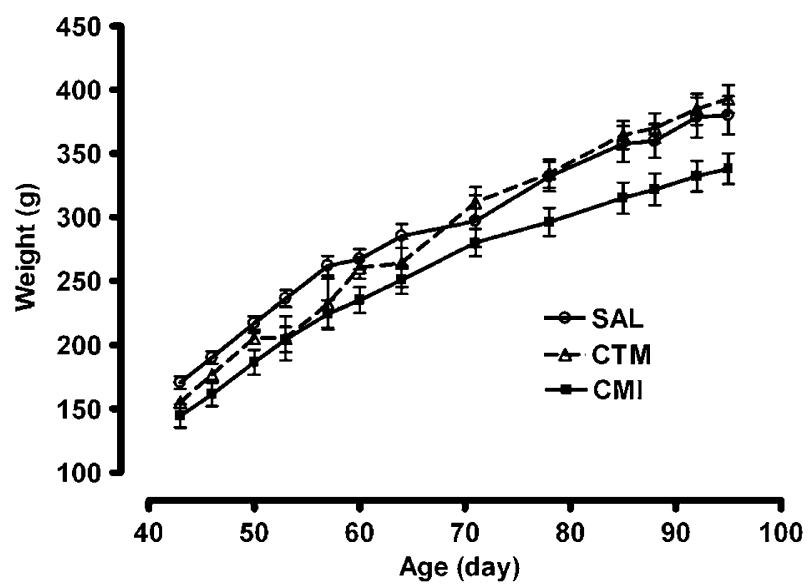

Figure 3 Effect of neonatal exposure to saline (SAL), citalopram (CTM), and clomipramine on body weight. Data represent the mean \pm SEM.

\section{Body Weight}

Rats were monitored for their body weight, and in all groups adult body weight increased steadily with age (ANOVA, $\mathrm{F}_{12,168}=362.13, P<0.001$ ). An effect of neonatal drug exposure (ANOVA, $\mathrm{F}_{2,14}=3.751, P=0.05$ ) and exposure by age interaction (ANOVA, $\mathrm{F}_{24,168}=2.353$, $P=0.001$ ) was observed. For example at PN 95, the mean body weights of control, citalopram- and clomipramineexposed rats were $379.8 \pm 14.8,392.6 \pm 10.7$, and $338.0 \pm$ $12.2 \mathrm{~g}$, respectively (Figure 3 ). Throughout adult life, there was a significant tendency only for clomipramine-exposed animals to have a lower body weight.

\section{Drug Concentration}

In order to ascertain that our drug exposure regime did not result in toxic blood or brain drug concentrations, samples of brain and serum taken after the last drug injection were analyzed. Serum and brain concentrations of citalopram, clomipramine, and its active metabolite desclomipramine were within experimental ranges obtained previously in adult rats. Furthermore, the serum concentrations were comparable within an order of magnitude to typical human serum concentrations after chronic administration of clinically active doses (Table 1).

\section{TPH, SERT, and NeuN Immunocytochemistry}

A significant decrease in the immunoreactivity of TPH was detected in the dorsal, as well as the median raphe of rats neonatally exposed to citalopram and, to a lesser extent, clomipramine at PN22 (Figure 4). The losses of CY3 fluorescent neurons were particularly striking in subregions of the DR that have been reported previously to contain projections to cortex (Jacobs and Azmitia, 1992). These voids in TPH expression are clearly evident along the midline subgroups, and are also detectable throughout the entire rostro-caudal extent of the DR neuraxis (arrows). This decrease appeared permanent, persisting through PN130 (Figure 5).

Given the likelihood that changes in the expression profiles of serotonergic cell bodies translate to terminal processes within raphe target sites, we examined the effects of neonatal citalopram on SERT immunoreactivity in select regions of the cortex which paralleled those observed in raphe on TPH. Specifically, SERT immunoreactivity was

Table I Drug and Metabolite Concentrations

\begin{tabular}{|c|c|c|c|c|c|}
\hline \multirow[b]{2}{*}{ Drug } & \multicolumn{3}{|c|}{ Serum (ng/ml) } & \multicolumn{2}{|c|}{ Brain (ng/g) } \\
\hline & Rat-PN2 I & Adult rat* & Human** & Rat-PN2 I & Adult rat* \\
\hline Clomipramine & $230 \pm 43$ & $208-416[1]$ & $100-250$ [3] & $2960 \pm 347$ & $4180-8360[1]$ \\
\hline Desclomipramine & $75 \pm 22$ & $408-816[1]$ & $50-200$ [3] & $1460 \pm 330$ & $2170-4340[1]$ \\
\hline
\end{tabular}

Data represent the mean \pm SEM of 4-5 samples/group. Reference concentrations obtained after chronic treatment with clomipramine (*15-30 mg/kg/day; $* * 1.4-$ $2.9 \mathrm{mg} / \mathrm{kg} / \mathrm{day}$ ) and citalopram (*10-20 mg/kg/day; ***3-0.6 mg/kg/day). [l]_(Friedman and Cooper, 1983), [2]—(Kugelberg et al, 200 I), [3]—(Vandel et al, I982), [4]-(Bjerkenstedt et al, 1985).

Bold values in table denote data obtained in our study. Regular format indicates values drawn from the literature. 

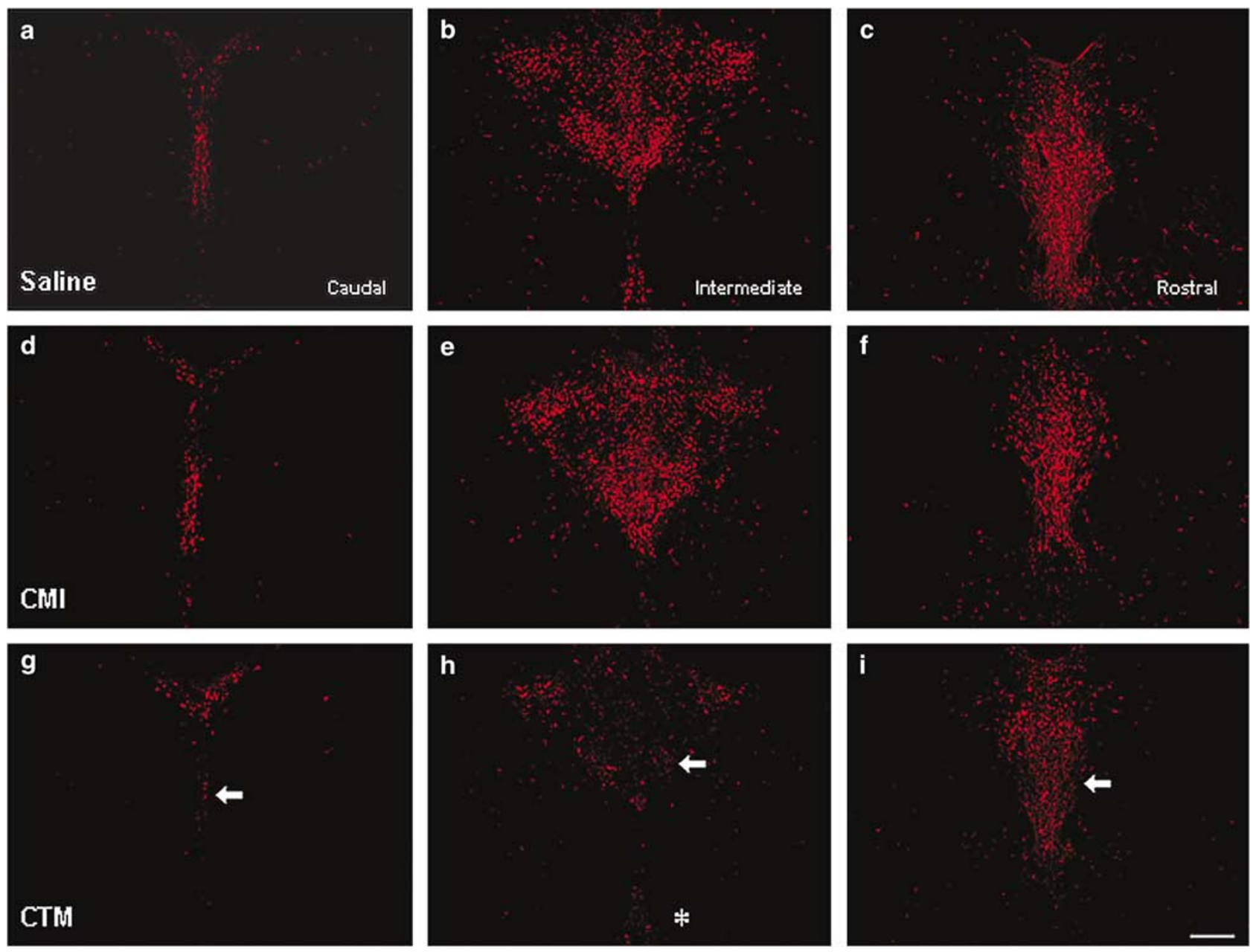

Figure 4 Representative photomicrographs through the DR show the caudal to rostral distribution of TPH-immunoreactive cells in untreated (a-c), clomipramine (CMl; $d-f)$-, and citalopram (CTM; g-i)-treated subjects at PN22. Note that reduced TPH expression was detected along the entire extent of the DR neuraxis (arrows), but was particularly evident within midline subregions. The median raphe, also depicted here, shows similar reductions in TPH immunoreactivity (asterisk). Scale bar $=100 \mu \mathrm{m}$.

decreased in the medial prefrontal cortex and primary somatosensory cortex in citalopram-treated rats (Table 2). This reduction was evident throughout all cortical layers and was observed within $24 \mathrm{~h}$ of the last drug treatment (PN22) (Figure 6). More specifically, citalopram-treated animals exhibited discontinuity of SERT-immunoreactive axons as compared to saline-treated animals (Figure $6 \mathrm{~d}-\mathrm{f}$ ). Global inspection of other cortical areas, such as the hippocampus, also revealed prominent reductions in the SERT-immunoreactive fiber network (data not shown). Moreover, the effects of citalopram on SERT fiber labeling were found to persist into adulthood-PN130 (Figure 7). Similar analysis of clomipramine-treated subjects revealed a nonsignificant effect of drug exposure on cortical SERT innervation; although it should be noted that a trend for decreased immunoreactivity was detected in some individual cases.

Initial examination at a light microscopic level revealed no obvious signs of gliosis, no apparent change in the packing density of cells, and no signs of shrinkage of somata or uneven Nissl staining (data not shown) to suggest that early SSRI exposure resulted in overt neuronal degeneration. To systematically determine whether the reduction in enzyme markers was the result of neuronal death, we also examined sections that were costained for both TPH and the neuron-specific marker, NeuN (Figure 8 and insets a, b). We obtained robust NeuN staining in sections of DR from rats exposed to citalopram (Figure $8 \mathrm{~b}$ ). NeuN staining was readily evident in neurons that were strongly and weakly labeled for TPH, as well as in somata that were TPH negative (Figure $8 \mathrm{a}$ ), suggesting no obvious neuronal death in the DR following citalopram treatment.

\section{DISCUSSION}

The present study demonstrates that neonatal exposure to a highly selective SSRI, citalopram, is at least as effective as neonatal exposure to the mixed action tricyclic antidepressant, clomipramine, at producing NADES. These effects were not due to increased blood or brain exposure to these antidepressants during early life because serum and brain 

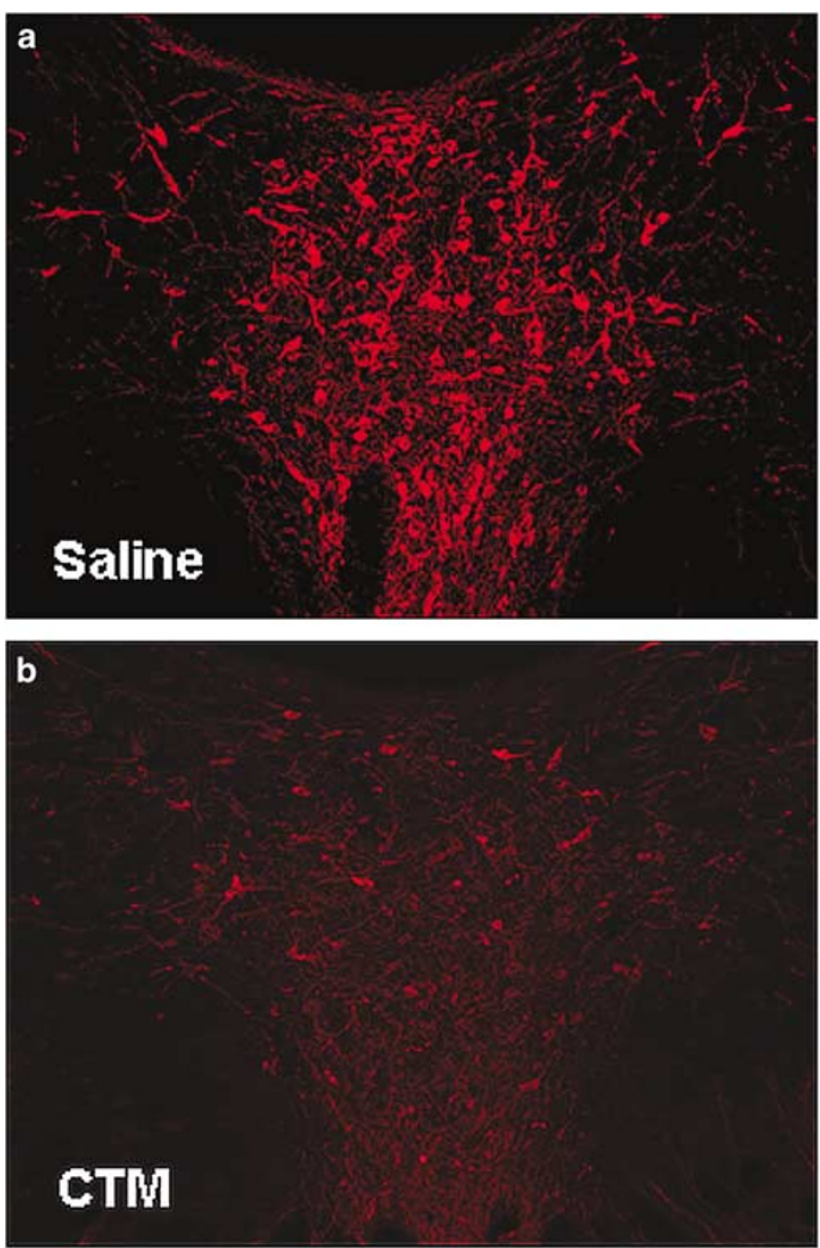

Figure 5 Representative photomicrographs of TPH immunoreactivity with $\mathrm{CY} 3$ in the DR nucleus of saline (a)- and citalopram (CTM; b)-treated (PN8-2I) rats at PNI30. drug/metabolite concentrations were similar to those achieved in adult treatment in rats. Moreover, the serum drug/metabolite concentrations achieved in the neonatal rats are quite similar to those achieved in humans at therapeutic doses of these drugs. It is noteworthy that throughout the adult life, only clomipramine-exposed animals had a lower body weight than saline-treated which is consistent with previous studies (Corner et al, 1980; De Boer et al, 1989), whereas there was no difference in body

Table 2 Effect of Neonatal SSRI Exposure on SERT Immunoreactivity

\begin{tabular}{llll}
\hline & Saline & CMI & CTM \\
\hline Somatosensory cortex & & & \\
Set I & Baseline & +7.62 & -34.21 \\
Set II & Baseline & -18.85 & -41.81 \\
Set III & Baseline & +18.09 & -35.55 \\
Set IV & Baseline & +19.22 & -39.81 \\
Mean & & $\mathbf{+ 6 . 5 2}$ & $-\mathbf{3 7 . 8 5}$ \\
& & & \\
Medial prefrontal cortex & & & \\
Set I & Baseline & +9.57 & -19.41 \\
Set II & Baseline & -29.34 & -22.01 \\
Set III & Baseline & -14.45 & -54.40 \\
Set IV & Baseline & +57.86 & -60.19 \\
Mean & & $\mathbf{+ 5 . 9 1}$ & $-\mathbf{3 9 . 0 0}$ \\
\hline
\end{tabular}

Data are representative sets of sections stained for SERT immunoreactivity. Citalopram diminished staining in somatosensory and medial prefrontal cortex to 62 and $61 \%$ of saline, respectively-Mann-Whitney $U, Z=-2.46,-2.46$, $P=0.014$ for citalopram vs saline, $n=4 /$ group.

Bold values in table denote data obtained in our study. Regular format indicates values drawn from the literature.
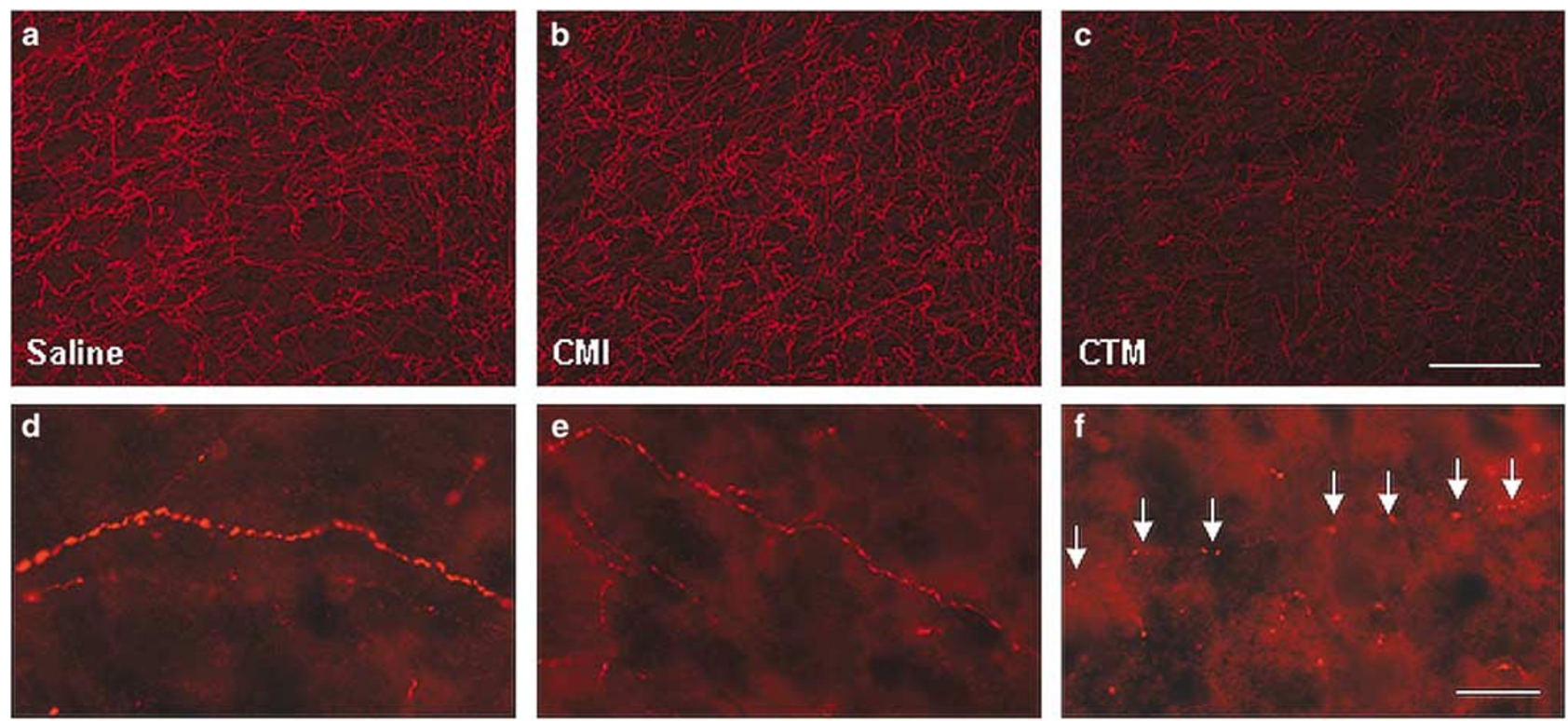

Figure 6 SERT expression in medial prefrontal cortex (layer II/III) of PN22 rats following saline (a), clomipramine (CMI; b), and citalopram (CTM; c) treatment from PN8 to PN2 I. (a-c) Illustrate immunoreactive fiber networks as visualized with CY3, while (d-f) demonstrate changes in the morphology of individual axons. Immediately following neonatal CTM treatment, the innervation density of SERT-positive processes is drastically reduced (c), and fibers exhibit a beaded appearance ( $\mathrm{f}$ ). Discontinuity of the axon is marked by a lack of immunolabeling within intervaricose segments. Arrows in (f) depict a trail of seemingly 'isolated' synaptic boutons. Scale bar: $a-c=100 \mu \mathrm{m} ; \mathrm{d}-\mathrm{f}=20 \mu \mathrm{m}$. 


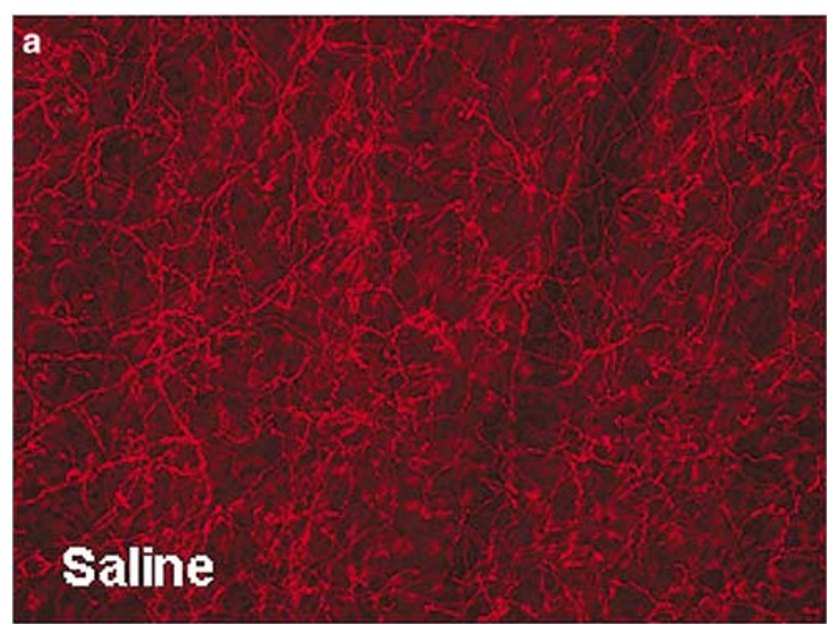

b CTM

Figure 7 Representative photomicrographs depict SERT terminal expression in the medial prefrontal cortex (layer II/III) of PNI30 rats following saline (a) and citalopram (CTM; b) treatment from PN8 to PN2I. $(\mathrm{a}, \mathrm{b})$ Illustrate immunoreactive fiber networks as visualized with CY3. Scale bar $=100 \mu \mathrm{m}$. weight between saline- and citalopram-exposed rats. Thus citalopram-exposed rats with no symptoms of malnutrition or abnormal physical signs exhibited abnormalities in adult behavior typical of NADES. Furthermore, our data indicates that neonatal exposure to citalopram or clomipramine produces profound changes in serotonergic circuitry including reduction in TPH immunoreactivity in dorsal and median raphe as well as SERT-immunoreactive fiber networks in cortical areas. These changes were detected $24 \mathrm{~h}$ after the last drug injection (PN22) and were found to persist into adulthood (PN130). Interestingly, NeuN staining revealed that, following citalopram treatment, DR neurons remain viable despite reductions in TPH expression, indicating an alteration of neuronal function rather than neuronal loss. Together, these data indicate that NADES is a specific consequence of serotonin transport inhibition during a critical period of development and that these behaviors are accompanied by profound changes in the serotonergic projection system with no apparent neurotoxicity.

It should be noted that the intensity of the reduction in immunohistochemical labeling of TPH was greater in citalopram-treated rats than in clomipramine-treated animals. Similarly, although reductions in SERT were apparent in both treatment groups, only the changes in citalopramtreated rats reached statistical significance. However, these appear to be quantitative rather than qualitative differences. This initial study employed single doses of citalopram and clomipramine chosen on the basis of literature reports. Notably, while the citalopram dose $(10 \mathrm{mg} / \mathrm{kg} /$ day $)$ is very near the $\mathrm{ED}_{50}$ for the in vivo activity of this drug, the dose of clomipramine $(30 \mathrm{mg} / \mathrm{kg} /$ day $)$ was chosen from the minimum effective dose (MED) reported previously to reliably induce NADES. This difference in the basis for dosing reflected our concerns that side effects, particularly inhibition of norepinephrine reuptake and antagonism of muscarinic cholinergic receptors, associated with this tricyclic antidepressant with limited selectivity might
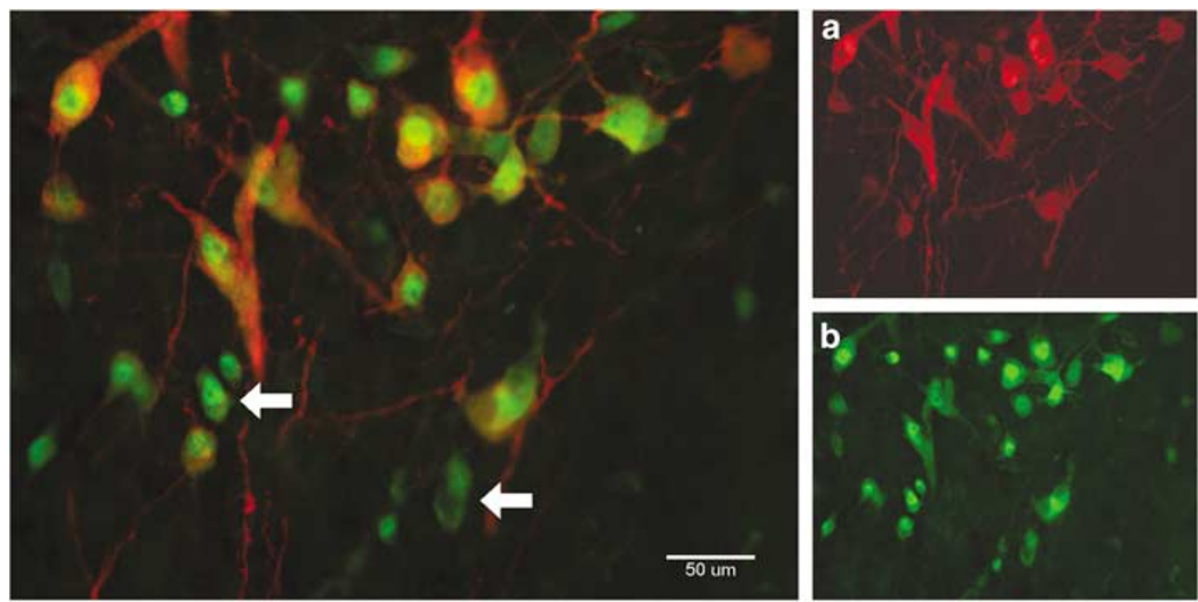

Figure 8 Photomicrograph taken at PN22 following neonatal citalopram treatment demonstrates TPH-labeled cells in DR that colocalize the neuronspecific marker, NeuN. Taken together with insets (a) and (b) of the same tissue section, which show single-labeled TPH (red, CY3) and viable NeuNpositive (green, CY2) cells, this figure illustrates that citalopram-related reductions in TPH are not due to cell death. As can be seen from surveys of individual cells in (a), treatment produces a nonuniform decrease in the expression of TPH. NeuN staining, however, remains prominent (arrows), despite these reductions. 
Table 3 Transporters and Receptors Affinities for Clomipramine and Citalopram

\begin{tabular}{|c|c|c|c|}
\hline \multirow[b]{2}{*}{ Receptor } & \multicolumn{2}{|c|}{$K_{\mathrm{i}}(\mathrm{nM})$} & \multirow[b]{2}{*}{ CMI/CTM $^{\mathrm{a}}$} \\
\hline & $\begin{array}{l}\text { Clomipramine } \\
\text { (CMI) }\end{array}$ & $\begin{array}{l}\text { Citalopram } \\
\text { (CTM) }\end{array}$ & \\
\hline SERT & 0.49 & 4.82 & 0.1 \\
\hline DAT & 2125 & None & 0.00001 \\
\hline NET & 40.3 & 8996 & 0.004 \\
\hline $5 \mathrm{HT}_{\mid \mathrm{A}}$ & None & None & - \\
\hline $5 \mathrm{HT}_{\mathrm{IB}}$ & None & None & - \\
\hline $5 \mathrm{HT}_{I D}$ & None & Negligible & - \\
\hline $5 \mathrm{HT}_{2}$ & $\mathrm{n} / \mathrm{a}$ & 3185 & $\mathrm{n} / \mathrm{a}$ \\
\hline $5 \mathrm{HT}_{2 \mathrm{~A}}$ & 20.5 & 5026 & 0.004 \\
\hline $5 \mathrm{HT}_{2 \mathrm{C}}$ & 46.2 & 1562 & 0.03 \\
\hline $5 \mathrm{HT}_{3}$ & 535 & Negligible & 0.00001 \\
\hline $5 \mathrm{HT}_{6}$ & 53.8 & $\mathrm{n} / \mathrm{a}$ & $\mathrm{n} / \mathrm{a}$ \\
\hline $5 \mathrm{HT}_{7}$ & 127 & $\mathrm{n} / \mathrm{a}$ & $\mathrm{n} / \mathrm{a}$ \\
\hline$M_{1}$ & $\begin{array}{c}\mathrm{n} / \mathrm{a}(\sim 183 \text { based } \\
\text { on imipramine) }\end{array}$ & 1430 & 0.1 \\
\hline$\alpha_{1}$ & 15.5 & None & 0.00001 \\
\hline$\alpha_{\text {la }}$ & 3.2 & None & 0.00001 \\
\hline$\alpha_{2}$ & none & None & - \\
\hline$\alpha_{2 a}$ & 525 & None & 0.00001 \\
\hline$D_{1}$ & 219 & $\mathrm{n} / \mathrm{a}$ & $\mathrm{n} / \mathrm{a}$ \\
\hline $\mathrm{D}_{2}$ & 120 & None & 0.00001 \\
\hline$D_{3}$ & 40 & None & 0.00001 \\
\hline
\end{tabular}

$K_{i}$ (inhibition constant) reflects the affinity of the drug for the transporter or receptor, that is, the lower the value the greater the affinity.

${ }^{a}<$ I means CMI more potent; $>$ I means CTM more potent. SERT, serotonin transporter; DAT, dopamine transporter; NET, noradrenaline transporter; none, no affinity; n/a, no data available. Data on drug affinities represent the mean value obtained from NIMH Psychoactive Drug Screening Program (PDSP) database (http://pdsp.cwru.edu/pdsp.asp).

confound our results (see Table 3 for a comparison of the binding affinities of these drugs). Further studies of the dose-response relationship for these drugs will be required to completely resolve this question.

This is certainly not the first study to evaluate changes in the serotonin system following neonatal antidepressant treatment. Previous studies have shown that following neonatal exposure to clomipramine, zimelidine, or desipramine, several brain regions in adult rats exhibit reductions in extracellular concentrations of serotonin (Hilakivi et al, 1987b; Feenstra et al, 1996; Vijayakumar and Meti, 1999). Moreover, early administration of clomipramine or Lu-10$134 \mathrm{C}$, a putative SSRI, produces a downregulation of DR SERT mRNA levels in adult rats (Hansen and Mikkelsen, 1998), and in some cases reduces the baseline firing rate of DR neurons (Kinney et al, 1997). However, it should be noted that these earlier studies used drugs that were either not entirely selective for the SERT (clomipramine, zimelidine) or whose specificity is not entirely clear (Lu-10-134C). Thus, antidepressant effects at various other sites (eg muscarinic cholinergic or $\alpha$ adrenergic receptors or other monoamine transporters) could not be excluded as contributors to NADES. By using citalopram, a wellestablished SSRI with little if any detected selectivity for any other recognition site, we can assert that NADES is specifically due to inhibition of the SERT during a developmentally critical period.

Although our data are in accord with these earlier findings, the critical difference between our study and others is that the current report is the first to link changes in neuronal expression at the cell body level with processes occurring at the most distal aspects of the neuron, that is, the cortical hemispheres. This approach has enabled us to track chemical relationships along the extent of the raphe projection in individual animals, and has more importantly permitted a correlation between cellular events and behavior. From these data, we can conclude that neonatal SSRI exposure (1) restructures regulatory pathways in the ascending serotonin projection and (2) elicits effects that are specific to inhibition of serotonin reuptake.

In addition to cohorts exposed to antidepressants as neonates, we tested the long-term effects of antidepressant exposure in adult rats. In contrast to the lasting effects of neonatal exposure, 40 or more days after the cessation of adult antidepressant treatment, no significant differences on any of the indices reported above were observed between animals exposed to 14 days of citalopram or clomipramine, even though the doses and route of administration were identical to that of neonatal animals (data not shown). These data demonstrate that the effects of exposure to citalopram or clomipramine on both behavior and immunohistochemical indices of serotonergic function are a specific consequence of exposure during a critical period of development.

It remains to be determined whether the effects of neonatal SSRI exposure on the rate-limiting serotonergic synthetic enzyme or transporter are causally related to the alterations in behavior produced by these treatments. Nonetheless, the present data offer valuable insight. Inasmuch as citalopram's effect on male sexual behavior and TPH and SERT immunoreactivity was very robust and clomipramine's was more modest, it can be suggested that these effects of neonatal antidepressant exposure are related and dependent on the individual substrate selectivity of the drug. Indeed, in addition to the SERT, clomipramine and its principal metabolite, desmethylclomipramine, have been shown to act on a number of central sites including the norepinephrine transporter (NET), and $\alpha_{1}$ adrenergic, $\mathrm{M}_{1}$ acetylcholine, and $\mathrm{H}_{1}$ histamine receptors (Table 3) (Hyttel, 1982).

Finally, the present studies do not address the neurobiological mechanism(s) by which early but not adult SSRI exposure effects lasting alteration in the expression of TPH and SERT. It can be logically argued that this response is due to an increase in extracellular serotonin concentrations during a developmentally critical period. However, the mechanism(s) by which increased extracellular serotonin regulates TPH and/or SERT is unclear. Moreover, additional studies of mRNA transcription and post-translational modification of these proteins will be required to determine whether the observed loss of immunoreactivity reflects reductions in protein expression or other modifications that interfere with antigen recognition. 
Finally, in view of recent reports of age-specific effects on antidepressants in humans, these data suggest that caution is warranted with regard to exposure to SSRIs during periods of rapid brain development. Inasmuch as the effects of antidepressants on brain and behavioral development seem to be related to their ability to inhibit the SERT, it is tempting to suggest that antidepressants with lower affinity for the SERT and greater affinity for the NET may be a safer treatment option during periods of rapid brain development. Likewise, antidepressant prototypes in development that target nonmonoaminergic sites (Skolnick et al, 2001) offer promise as alternative therapies.

\section{ACKNOWLEDGEMENTS}

We thank Ms LaShondra Williams for excellent technical assistance. We have no competing interests relevant to this manuscript. This publication was supported in part by research funds from the Center of Psychiatric Neuroscience at the University of Mississippi Medical Center, which is supported by NIH Grant Number RR-P20 RR17701 from the Institutional Developmental Award (IDeA) Program of the National Center for Research Resources.

\section{REFERENCES}

Ansorge MS, Zhou M, Lira A, Hen R, Gingrich JA (2004). Early-life blockade of the 5-HT transporter alters emotional behavior in adult mice. Science 306: 879-881.

Azmitia EC (2001). Modern views on an ancient chemical: serotonin effects on cell proliferation, maturation, and apoptosis. Brain Res Bull 56: 413-424.

Bjerkenstedt L, Flyckt L, Overo KF, Lingjaerde O (1985). Relationship between clinical effects, serum drug concentration and serotonin uptake inhibition in depressed patients treated with citalopram. A double-blind comparison of three dose levels. Eur J Clin Pharmacol 28: 553-557.

Casper RC, Fleisher BE, Lee-Ancajas JC, Gilles A, Gaylor E, DeBattista A et al (2003). Follow-up of children of depressed mothers exposed or not exposed to antidepressant drugs during pregnancy. J Pediatr 142: 402-408.

Cohen LS, Nonacs R, Viguera AC, Reminick A (2004). Diagnosis and treatment of depression during pregnancy. CNS Spectr 9: 209-216.

Corner MA, Mirmiran M, Bour HL, Boer GJ, van de Poll NE, van Oyen HG et al (1980). Does rapid-eye-movement sleep play a role in brain development? Prog Brain Res 53: 347-356.

Costei AM, Kozer E, Ho T, Ito S, Koren G (2002). Perinatal outcome following third trimester exposure to paroxetine. Arch Pediatr Adolesc Med 156: 1129-1132.

De Boer S, Mirmiran M, Van Haaren F, Louwerse A, van de Poll NE (1989). Neurobehavioral teratogenic effects of clomipramine and alpha-methyldopa. Neurotoxicol Teratol 11: 77-84.

Dobbing J (1974). The later development of the brain and its vulnerability. In Davis JA, Dobbing J (eds). Scientific Foundations of Paediatrics. W.B. Saunders Co.: Philadelphia, PA. pp 565-577.

Feenstra MG, van Galen H, Te Riele PJ, Botterblom MH, Mirmiran M (1996). Decreased hypothalamic serotonin levels in adult rats treated neonatally with clomipramine. Pharmacol Biochem Behav 55: 647-652.

Friedman E, Cooper TB (1983). Pharmacokinetics of chlorimipramine and its demethylated metabolite in blood and brain regions of rats treated acutely and chronically with chlorimipramine. J Pharmacol Exp Ther 225: 387-390.
Hansen HH, Mikkelsen JD (1998). Long-term effects on serotonin transporter mRNA expression of chronic neonatal exposure to a serotonin reuptake inhibitor. Eur J Pharmacol 352: 307-315.

Hansen HH, Sanchez C, Meier E (1997). Neonatal administration of the selective serotonin reuptake inhibitor Lu 10-134-C increases forced swimming-induced immobility in adult rats: a putative animal model of depression? J Pharmacol Exp Ther 283: 1333-1341.

Hansson SR, Mezey E, Hoffman BJ (1998). Serotonin transporter messenger RNA in the developing rat brain: early expression in serotonergic neurons and transient expression in non-serotonergic neurons. Neuroscience 83: 1185-1201.

Hartley P, Neill D, Hagler M, Kors D, Vogel G (1990). Procedureand age-dependent hyperactivity in a new animal model of endogenous depression. Neurosci Biobehav Rev 14: 69-72.

Hendrick V, Stowe ZN, Altshuler LL, Hwang S, Lee E, Haynes D (2003). Placental passage of antidepressant medications. Am J Psychiatry 160: 993-996.

Hilakivi LA, Hilakivi I (1987). Increased adult behavioral 'despair' in rats neonatally exposed to desipramine or zimeldine: an animal model of depression? Pharmacol Biochem Behav 28: 367-369.

Hilakivi LA, Hilakivi I, Ahtee L, Haikala H, Attila M (1987a). Effect of neonatal nomifensine exposure on adult behavior and brain monoamines in rats. J Neural Transm 70: 99-116.

Hilakivi LA, Sinclair JD, Hilakivi IT (1984). Effects of neonatal treatment with clomipramine on adult ethanol related behavior in the rat. Brain Res 317: 129-132.

Hilakivi LA, Stenberg D, Sinclair JD, Kiianmaa K (1987b). Neonatal desipramine or zimeldine treatment causes long-lasting changes in brain monoaminergic systems and alcohol related behavior in rats. Psychopharmacology 91: 403-409.

Holmes A, Murphy DL, Crawley JN (2003). Abnormal behavioral phenotypes of serotonin transporter knockout mice: parallels with human anxiety and depression. Biol Psychiatry 54: 953-959.

Hostetter A, Ritchie JC, Stowe ZN (2000). Amniotic fluid and umbilical cord blood concentrations of antidepressants in three women. Biol Psychiatry 48: 1032-1034.

Hyttel J (1982). Citalopram - pharmacological profile of a specific serotonin uptake inhibitor with antidepressant activity. Prog Neuropsychopharmacol Biol Psychiatry 6: 277-295.

Ivgy-May N, Tamir H, Gershon MD (1994). Synaptic properties of serotonergic growth cones in developing rat brain. J Neurosci 14: 1011-1029.

Jacobs BL, Azmitia EC (1992). Structure and function of the brain serotonin system. Physiol Rev 72: 165-229.

Johnston MV, Coyle JT (1980). Ontogeny of neurochemical markers for noradrenergic, GABAergic, and cholinergic neurons in neocortex lesioned with methylazoxymethanol acetate. J Neurochem 34: 1429-1441.

Kinney GG, Vogel GW, Feng P (1997). Decreased dorsal raphe nucleus neuronal activity in adult chloral hydrate anesthetized rats following neonatal clomipramine treatment: implications for endogenous depression. Brain Res 756: 68-75.

Kugelberg FC, Apelqvist G, Carlsson B, Ahlner J, Bengtsson F (2001). In vivo steady-state pharmacokinetic outcome following clinical and toxic doses of racemic citalopram to rats. $\mathrm{Br} J$ Pharmacol 132: 1683-1690.

Laine K, Heikkinen T, Ekblad U, Kero P (2003). Effects of exposure to selective serotonin reuptake inhibitors during pregnancy on serotonergic symptoms in newborns and cord blood monoamine and prolactin concentrations. Arch Gen Psychiatry 60: 720-726.

Lauder JM (1990). Ontogeny of the serotonergic system in the rat: serotonin as a developmental signal. Ann N Y Acad Sci 600: 297-313; discussion 314.

Lauder JM, Bloom FE (1974). Ontogeny of monoamine neurons in the locus coeruleus, Raphe nuclei and substantia nigra of the rat. I. Cell differentiation. J Comp Neurol 155: 469-481. 
Lauder JM, Bloom FE (1975). Ontogeny of monoamine neurons in the locus coeruleus, raphe nuclei and substantia nigra of the rat. II. Synaptogenesis. J Comp Neurol 163: 251-264.

Li Q, Wichems C, Heils A, Lesch KP, Murphy DL (2000). Reduction in the density and expression, but not G-protein coupling, of serotonin receptors (5-HT1A) in 5-HT transporter knockout mice: gender and brain region differences. J Neurosci 20: 7888-7895.

Li Q, Wichems CH, Ma L, Van de Kar LD, Garcia F, Murphy DL (2003). Brain region-specific alterations of 5-HT2A and 5-HT2C receptors in serotonin transporter knockout mice. J Neurochem 84: $1256-1265$.

Lira A, Zhou M, Castanon N, Ansorge MS, Gordon JA, Francis JH et al (2003). Altered depression-related behaviors and functional changes in the dorsal raphe nucleus of serotonin transporterdeficient mice. Biol Psychiatry 54: 960-971.

Mirmiran M, Scholtens J, van de Poll NE, Uylings HB, van der Gugten J, Boer GJ (1983). Effects of experimental suppression of active (REM) sleep during early development upon adult brain and behavior in the rat. Brain Res 283: 277-286.

Mirmiran M, van de Poll NE, Corner MA, van Oyen HG, Bour HL (1981). Suppression of active sleep by chronic treatment with chlorimipramine during early postnatal development: effects upon adult sleep and behavior in the rat. Brain Res 204: 129-146.

Nordeng H, Lindemann R, Perminov KV, Reikvam A (2001). Neonatal withdrawal syndrome after in utero exposure to selective serotonin reuptake inhibitors. Acta Paediatr 90: 288-291.

Nulman I, Rovet J, Stewart DE, Wolpin J, Pace-Asciak P, Shuhaiber $S$ et al (2002). Child development following exposure to tricyclic antidepressants or fluoxetine throughout fetal life: a prospective, controlled study. Am J Psychiatry 159: 1889-1895.

Ogawa T, Mikuni M, Kuroda Y, Muneoka K, Mori KJ, Takahashi K (1994). Effects of the altered serotonergic signalling by neonatal treatment with 5, 7-dihydroxytryptamine, ritanserin or clomipramine on the adrenocortical stress response and the glucocorticoid receptor binding in the hippocampus in adult rats. J Neural Transm Gen Sect 96: 113-123.

Prathiba J, Kumar KB, Karanth KS (1998). Hyperactivity of hypothalamic pituitary axis in neonatal clomipramine model of depression. J Neural Transm 105: 1335-1339.

Rind HB, Russo AF, Whittemore SR (2000). Developmental regulation of tryptophan hydroxylase messenger RNA expression and enzyme activity in the raphe and its target fields. Neuroscience 101: 665-677.
Sanz EJ, De-las-Cuevas C, Kiuru A, Bate A, Edwards R (2005). Selective serotonin reuptake inhibitors in pregnant women and neonatal withdrawal syndrome: a database analysis. Lancet 365: 482-487.

Skolnick P, Legutko B, Li X, Bymaster FP (2001). Current perspectives on the development of non-biogenic amine-based antidepressants. Pharmacol Res 43: 411-423.

Sodhi MS, Sanders-Bush E (2004). Serotonin and brain development. Int Rev Neurobiol 59: 111-174.

Vandel B, Vandel S, Jounet JM, Allers G, Volmat R (1982). Relationship between the plasma concentration of clomipramine and desmethylclomipramine in depressive patients and the clinical response. Eur J Clin Pharmacol 22: 15-20.

Verney C, Lebrand C, Gaspar P (2002). Changing distribution of monoaminergic markers in the developing human cerebral cortex with special emphasis on the serotonin transporter. Anat Rec 267: 87-93.

Vijayakumar M, Meti BL (1999). Alterations in the levels of monoamines in discrete brain regions of clomipramine-induced animal model of endogenous depression. Neurochem Res 24: 345-349.

Whitaker-Azmitia PM (1991). Role of serotonin and other neurotransmitter receptors in brain development: basis for developmental pharmacology. Pharmacol Rev 43: 553-561.

Wisner KL, Zarin DA, Holmboe ES, Appelbaum PS, Gelenberg AJ, Leonard HL et al (2000). Risk-benefit decision making for treatment of depression during pregnancy. Am J Psychiatry 157: 1933-1940.

Xu Y, Sari Y, Zhou FC (2004). Selective serotonin reuptake inhibitor disrupts organization of thalamocortical somatosensory barrels during development. Brain Res Dev Brain Res 150: 151-161.

Yannielli PC, Kargieman L, Gregoretti L, Cardinali DP (1999). Effects of neonatal clomipramine treatment on locomotor activity, anxiety-related behavior and serotonin turnover in Syrian hamsters. Neuropsychobiology 39: 200-206.

Yavari P, Vogel GW, Neill DB (1993). Decreased raphe unit activity in a rat model of endogenous depression. Brain Res 611: 31-36.

Zeskind PS, Stephens LE (2004). Maternal selective serotonin reuptake inhibitor use during pregnancy and newborn neurobehavior. Pediatrics 113: 368-375.

Zhou FC, Sari Y, Zhang JK (2000). Expression of serotonin transporter protein in developing rat brain. Brain Res Dev Brain Res 119: 33-45. 